\title{
Review: assumed properties of child sexual abuse are not borne out in college student samples
}

\author{
Rind B, Tromovitch P,Bauserman R. A meta-analytic examination of assumed properties of child sexual abuse using college samples. \\ Psychol Bull 1998 Jul;124:22-53.
}

\section{Question}

In college students with a history of child sexual abuse (CSA), what is the effect of CSA on psychological adjustment with regard to causality, pervasiveness, intensity, and gender equivalence?

\section{Data sources}

Studies were identified by searching PsycLIT (1974-95), Sociological Abstracts (1974-95), PsycINFO (1967-95), Dissertation Abstracts (to 1995), and ERIC (1966-95) using the terms adjustment, effect, college, undergraduate, sex abuse, child, and adult. Reference lists of obtained studies were also reviewed.

\section{Study selection}

Studies were selected if they included samples of college students for whom separate measures were available and contained data on reactions to CSA or self reported effects of CSA. Studies were also included in analyses of psychological correlates of CSA if they included a control group of students with no CSA experiences, provided data on $\geqslant 1$ of 18 psychological symptoms, and provided sufficient data to calculate $\geqslant 1$ effect size.

\section{Data extraction}

Data were extracted on psychological adjustment (symptoms), sex of participants, definition of CSA, reaction to CSA, self reported effects, and family environment.

\section{Main results}

59 studies met the inclusion criteria and included 35703 participants (62\% women) providing 54 student samples for calculating 54 sample level effect sizes and 214 symptom level effect sizes. Effect size data for psychological adjustment were based on 15824 respondents. Meta-analysis of the sample level effect sizes showed that students with CSA were slightly less well adjusted than control group students (mean weighted effect size $0.09,95 \%$ CI 0.08 to 0.11 ). At the symptom level, the effect size estimates ranged from 0.04 to 0.13 for the 18 psychological symptoms; all but 1 (locus of control) showed that students with CSA were less well adjusted than control group students. The effect sizes, however, were small indicating a weak association between CSA and psychological adjustment with symptoms being neither pervasive nor intense. Retrospectively recalled self reported reactions to CSA (data from 15 studies) differed between men and women: $72 \%$ of women's experiences were reported as negative compared with $33 \%$ of men's experiences. A meta-analysis of self reported effects of CSA combining 4 effect sizes gave an unbiased effect size estimate of 0.22 (CI 0.15 to 0.28 ) showing that women reported more negative effects than men. Further meta-analysis was done based on 14 samples that examined the potential confounding effects of family environment. For 59\% of the symptoms examined, the statistically significant CSA adjustment association became non-significant after controlling for family environment.

\section{Conclusions}

Among college students an association exists between a history of child sexual abuse and poorer psychological adjustment, but the magnitude of the association is small. Family environment is confounded with child sexual abuse and is a stronger predictor of poor adjustment than is child sexual abuse. A gender difference also exists, with women reacting more negatively to the experience than men.

Source of funding: No external funding.

For correspondence: Dr B Rind, Department of Psychology, Temple University, Philadelphia, PA 19122, USA.Fax +1215204 5539

\section{Commentary}

Few topics in psychology generate as much controversy as the issue of CSA. The meta-analytic review by Rind et al tackles one of the most contentious and crucial issues in this field: the nature and strength of the relation between CSA and psychological adjustment.

The authors have done a comprehensive meta-analysis using samples of college students. Their finding that college students with a history of CSA were, on average, only slightly less well adjusted than controls without a history of CSA, is encouraging information for people who have experienced sexual abuse in childhood. Nevertheless, this conclusion cannot be generalised to all people with a history of CSA. Rind et al highlight the similarities between sexually abused people in college and national samples. However, college students still represent a relatively special spectrum of the popula- tion in their sociodemographic characteristics. In addition, although 59 studies were included in the overall review, any one symptom was assessed in about $20 \%$ of the individual studies.

This meta-analysis has several strengths, including the use of explicit criteria for study selection, data extraction by 2 independent raters, and sophisticated assessment of the variability in sample level effect sizes. However, the review did not include an assessment of the methodological quality of the original studies. Such aspects as design, sampling technique, and response rate in the studies are essential aspects to consider, otherwise studies of varying quality are grouped together and given equal weight. ${ }^{1}$ In addition, the issue of CSA causing harm cannot be examined in cross sectional surveys; only longitudinal studies can investigate the causal role of CSA. Infor- mation about the design of the individual studies is therefore important in interpreting the results.

Rind et al conclude that CSA "does not cause intense harm on a pervasive basis" in the college population but, without taking into account the quality of the individual studies, this conclusion is premature. The findings of this review suggest that CSA may not be associated with poor psychological functioning generally in college students, but further research is necessary before definitive conclusions can be drawn.

Harriet MacMillan, MD, MSc, FRCP Hamilton Health Sciences Corporation Hamilton, Ontario, Canada

1 Lewis G, Churchill R, Hotopf M. Systematic reviews and meta-analysis. Psychol Med 1997;27:3-7. 